\title{
Telemedicine.......the future
}

\section{Editorial}

Increasingly amazed we with the ability to simplification and miniaturization of surveillance systems biological signals, this miniaturization is not only capable of allowing smaller sensors, but these are suitable to use wireless methods to transfer the distance information that is using telemedicine techniques. We can also, access to a group of sensors that transmit data more varied as pulse Oximetry to ECG pattern or levels of blood glucose. The ultimate goal of these systems is the ability to warn the patient that something is wrong and you should consult or even health programs and through artificial intelligence services themselves, know that certain patients are experiencing an evolution that is far from what is considered in the usual.

If a patient is able to use technologies that address their health, without having to frequently, go to health services, the economic costs definitely decrease, but also the patient may experience a sense of reduced dependence on health services. On the other hand the transmission of signals, could lead to having a large number of data, biological signals transmitted at low cost and with techniques of unconventional as nonlinear dynamic mathematical analysis to detect very early, a patient for example has an exacerbation of chronic obstructive pulmonary disease or that you must set the parameters of your CPAP, etc. The future is intended for systems that are fully
Volume 2 Issue 2 - 2015

Jose Bruno De Lema

High Complexity Foundation, Spain

Correspondence: Jose Bruno De Lema, High Complexity Foundation, Paseo de la montana \# 14 bajo. granollers 08402, Pereira, Barcelona, Spain, Email delemalarre@gmail.com

Received: February 24, 20I5 | Published: February 28, 2015

portable with an internal power supply and a wireless transmitter, which also taller than a signal and can even adjust other processing equipment. But that is yet to come.

\section{Acknowledgements}

None.

\section{Conflict of interest}

The author declares no conflict of interest. 